\title{
The case for a multi-channel polarization sensitive LIDAR for investigation of insolation-driven ices and atmospheres
}

\author{
Planetary Science Decadal Survey White Paper
}

Submitted July 10, 2020

Authors:

Adrian J. Brown, Plancius Research, MD adrian.j.brown@nasa.gov

Gorden Videen, Space Science Institute Evgenij Zubko, Kyung Hee University S. Korea Nicholas Heavens, Space Science Institute Nicole-Jeanne Schlegel, Jet Propulsion Lab Patricio Becerra, University of Bern, Schweiz Young-Jun Choi, KASI, South Korea Colin R. Meyer, Dartmouth College Tanya N. Harrison, Outer Space Institute Paul Hayne, University of Colorado Boulder Rachel W. Obbard, SETI Institute

Tim Michaels, SETI Institute Michael J. Wolff, Space Science Institute Scott Guzewich, NASA GSFC Yongxiang $\mathrm{Hu}, \mathrm{NASA}$ LARC

Claire Newman, Aeolis Research

Christian J. Grund

Chae Kyung Sim, Kyung Hee Uni., S. Korea

Peter B. Buhler, Jet Propulsion Lab

Margaret E. Landis, CU LASP

Timothy J. Stubbs, NASA GSFC

Aymeric Spiga, Sorbonne Uni., LMD, France

Devanshu Jha, MVJCE, India

\section{Signatories:}

Shane Byrne, LPL University of Arizona

Serina Diniega, Jet Propulsion Lab

Michael Mishchenko, NASA GISS

Sungsoo S. Kim, Kyung Hee Uni., S. Korea

Susan Conway, University of Nantes, France

Ken Herkenhoff, USGS Astrogeology

Michael Mischna, Jet Propulsion Lab

Anthony Colaprete, NASA Ames

Minsup Jeong, KASI, S. Korea

Isaac Smith, York University, Canada and

Planetary Science Institute, Colorado

Matthew R. Perry, Planetary Science Institute

John E. Moores, York University, Canada

Christine S. Hvidberg, Niels Bohr Inst., Denmark

Jonathan A. R. Rall, Off. of Chf Sci., NASA HQ

Sylvain Piqueux, Jet Propulsion Lab

Robert Lillis, SSL UC Berkeley

Leslie Tamppari, Jet Propulsion Lab

Wendy Calvin, University of Nevada

Jennifer Hanley, Lowell Observatory

Nathaniel Putzig, Planetary Science Institute

Ali M. Bramson, Purdue University

Bonnie Meineke, Ball Aerospace

Michael Veto, Ball Aerospace

Jack Holt, University of Arizona

Bryana L. Henderson, Jet Propulsion Lab

Lori K. Fenton, SETI Institute

Alain Khayat, NASA GSFC/UMD

Tim McConnochie, UMD/Space Science Inst.

Timothy N. Titus, USGS Astrogeology

\section{"There once was a LIDAR with Polarized Holes, Made to observe Mars' Mysterious Poles, It measured Volatiles and Ices ... and used Multispectral Devices ... And met many of our Mars Climate Goals."}

- Anon, 2020 
Key point of this white paper: All LIDAR instruments are not the same, and advancement of LIDAR technology requires an ongoing interest and demand from the community to foster further development of the required components. The purpose of this white paper is to make the decadal survey panel aware of the need for further technical development, and the potential payoff of investing experimental time, money and thought into the next generation of LIDARs. Technologies for development: We advocate for future development of LIDAR technologies to measure the polarization state of the reflected light at selected multiple wavelengths, chosen according to the species of interest (e.g., $\mathrm{H}_{2} \mathrm{O}$ and $\mathrm{CO}_{2}$ in the Martian setting).

Key scientific questions: In the coming decade, dollars spent on these LIDAR technologies will go towards addressing key climate questions on Mars and other rocky bodies, particularly those with seasonally changing (i.e. insolation driven) plumes of multiple icy volatiles such as Mars, Enceladus, Triton, or Pluto, and insolation-driven dust lifting, such as cometary bodies and the Moon. We will show from examining past Martian and terrestrial lidars that orbital and landed LIDARs can be effective for producing new insights into insolation-driven processes in current planetary climate on several bodies, beyond that available to our current fleet of largely passive instruments on planetary missions.

\section{Preamble and Science Themes}

This white paper is intended to make the decadal survey panel aware of the type and amount of information about the present day Martian climate that would be acquired by a multiple-wavelength, active, near-infrared (NIR) LIDAR instrument. This instrument would measure the reflected intensity and polarization characteristics of backscattered radiation from the surface via the atmosphere, such as that described by Brown et al. [1]. Science output from this type of dataset would address the following three major science themes:

Science Theme 1. Surface Global, night and day mapping of $\mathrm{H}_{2} \mathrm{O}$ and $\mathrm{CO}_{2}$ surface ice, Science Theme 2. Ice Clouds: Unambiguous discrimination and seasonal mapping of $\mathrm{CO}_{2}$ and $\mathrm{H}_{2} \mathrm{O}$ ice clouds

Science Theme 3. Dust Aerosols: Inference of dust grain shapes and size distributions from multiwavelength polarization measurements.

Knowledge generated from such an instrument has the potential to fundamentally shift our understanding of modern-day Martian volatile transport and deposition. As a bonus, this lidar would permit a continuation of the MOLA geodetic mapping of Mars to a point where a global network suitable for registration of high resolution images (e.g. HiRISE).

\section{Martian Example Case Study LIDAR - ASPEN}

Our present understanding of the sublimation of surface $\mathrm{H}_{2} \mathrm{O}$ and $\mathrm{CO}_{2}$ ices and related atmospheric changes on Mars is the result of recent polewide and seasonal studies of springtime recession using the Mars Climate Sounder [2], CRISM [3], and MARCI [4] 
instruments on MRO, the OMEGA instrument on Mars Express [5-6], the THEMIS instrument on 2001 Mars Odyssey [7] and the TES instrument on Mars Global Surveyor [8]. These investigations have steadily advanced our understanding of major polar processes. However, the observations of the spatially localized springtime recession phenomena, such as geysers (gas/dust jets, which are inferred, and have not been directly observed) [9], and observations of the asymmetric retraction of the seasonal cap $[6,10]$ lead us to ask a key scientific question - what role does spatially localized and temporally intermittent deposition of ices and dust during fall and winter [10] play in the annual $\mathrm{CO}_{2}$ and $\mathrm{H}_{2} \mathrm{O}$ cycles that are instrumental the climate of modern-day Mars? In the remainder of this white paper, we discuss an instrument optimized for Martian conditions called "Atmospheric/Surface Polarization Experiment at Nighttime" (ASPEN) [1], designed to directly address the role of ice and dust deposition in Martian climate.

Instrument Specs. ASPEN would be a multi-wavelength, altitude-resolved, active, near-infrared (NIR: covering 1.52-1.59 $\mu \mathrm{m}$ ) instrument to measure the reflected intensity and polarization of backscattered radiation from planetary surfaces and atmospheres. The currently envisioned spacecraft instrument utilizes multiple diode lasers, each operable at a different wavelength, amplified by a fiber laser stage (commonly referred to as a master-oscillator power-amplifier or MOPA). The laser would operate in a high repetition $(10 \mathrm{kHz})$ low pulse energy $(40 \mu \mathrm{J})$ configuration. The receiver side will consist of a telescope coupled to an indium gallium arsenide (InGaAs) high dynamic range avalanche photo detector (APD).

Martian Operations. As currently envisioned, the ASPEN instrument would operate as a line profile instrument, in a similar manner to the MOLA lidar. The instrument is best suited for an MGS or MRO-type 250-320 km elliptical/circular orbit but could also operate in an elliptical orbit with reduced sensitivity during apoapsis. For optimized polar measurements, orbital inclination should be between $85^{\circ}$ and $92.8^{\circ}$. An elliptical orbit such as that mentioned in the recent MSO SAG document would allow lidar-occultation measurements of the atmosphere, allowing the atmosphere to be viewed 'side on', thus enabling profile measurements of $\mathrm{CO}_{2}, \mathrm{H}_{2} \mathrm{O}$ ice and vapor in the Martian atmosphere. Preliminary instrument performance calculations of common measurement scenarios for the MOPA laser ASPEN instrument estimate the surface spot size at $\sim 25 \mathrm{~m}$ on the surface and a horizontal resolution of $\sim 275 \mathrm{~m}$ (similar to MOLA which was $\sim 330 \mathrm{~m}$ ).

Multi-wavelength. In order to take advantage of the tremendous research and development that has gone into lasers and fiber optic components that operate in the near-IR by the telecommunications industry in recent years, the instrument will operate at wavelengths between 1.52 and $1.59 \mu \mathrm{m}$. These wavelengths are ideally suited to 
discriminate $\mathrm{CO}_{2}$ and $\mathrm{H}_{2} \mathrm{O}$ ices and vapor using the differential absorption lidar (DIAL) technique originally developed for terrestrial remote sensing [26, 27].

The Mars Science community has previously recognized the need for an ASPEN-type instrument. The need for actively scanning laser sensors that operate over a range of frequencies was acknowledged in the 2006 Solar System Exploration Roadmap [11] (page 108). In addition, the 2013 Mars Science Orbiter Science Analysis Group (MSO SAG) report stated that a "multibeam lidar" similar to the LOLA on LRO, and inheriting many aspects from the CALIPSO LIDAR, would "resolve optically dense atmospheric phenomena" and "significantly constrain seasonal mass budgets". In essence, it was thought to be an ideal instrument for a "2013 MSO mission" [12].

Key Science: Previous instruments have given glimpses of cloud and surface ice activity on Mars, but no previous Martian orbital instrument has been able to simultaneously address the following science measurements, which ASPEN would:

a.) Detect clouds up to $100 \mathrm{~km}$ above the Martian surface during night and day;

b.) Discriminate between $\mathrm{H}_{2} \mathrm{O}$ and $\mathrm{CO}_{2}$ ice and dust on the surface and in aerosols in the atmosphere in both polar day and night [13];

c.) Map cloud structure using lidar backscatter and depolarization;

d.) Map large-grained (path length up to $30 \mathrm{~cm}$ ) $\mathrm{CO}_{2}$ slab ice in the polar night, which is uniquely Martian and is extremely poorly understood [14];

e.) Determine whether the $\mathrm{H}_{2} \mathrm{O}$ ice signature in the southern polar trough system is due to cloud [15] or surface ice [16];

f.) Monitor 'cold spot' activity during the polar night and determine whether these enigmatic features are due to $\mathrm{CO}_{2}$ clouds, precipitation or surface ice $[17,18]$;

g.) Monitor night and day gas/dust jet (geyser) activity within the 'Cryptic Region' (which have not yet been observed "in action") in southern late winter and early spring and determine what amount of solar energy is required for them to be active [8,19]; h.) Uniquely identify cloud types and platelet/grain orientation, in order to confirm the presence and structure of convective $\mathrm{CO}_{2}$ cloud towers, a potentially critical part of the polar night dynamics and energy partitioning [20];

i.) Atmospheric column dust optical depths when instrument is in operation $[21,22]$.

j.) Monitor the spring and summertime retreating polar caps for signs of sediment flows and possibly even geysers caused by subliming $\mathrm{CO}_{2}$ ice. This type of activity has already been suggested to cause substantial changes mid-latitude gullies [23] and on dunefields [24].

k.) Address questions of spatial extent (locality and 'deep transport') of Martian cloud structure, which is anticipated to be on the order of $1 \mathrm{~km}$ width and is crucial to understanding differences between terrestrial and Martian mesospheric dynamics [25]. i.) conduct a sensitive global (daytime and nighttime) search for outbursts of Martian methane down to $2 \mathrm{ppb}$ with relative errors of $0.5 \%$ [26,27]. 


\section{Instrument Concept and Background}

\subsection{A conceptual model for energy transport in the Martian climate and why a lidar is essential to reveal it}

Our understanding of the modern day Martian climate is based on relatively easy to visualize energy transport concepts. One can envisage the energy within the polar caps as governed by the latent heat of the ice in the caps, and can track the flow of ice from the caps to the atmosphere and mid latitudes during summer. These processes can be observed as the springtime recession of the seasonal cap, as the stored energy of the ice is broken up and moves into the atmosphere and the mid latitudes. Energy transport in the Martian climate has been tracked by telescopes and subsequent orbital instruments.

During winter, the reverse process is less certain, mainly because it can no longer be directly observed due to the onset of the polar hood and polar night [3]. It is during this time that the energy of the cap, like ice blocks in an igloo, is stored away again in the form of $\mathrm{CO}_{2}$ and $\mathrm{H}_{2} \mathrm{O}$ ice in the winter cap. However, this process is not well understood at all - we have some clues in the southern pole due to supercool spots that have been associated with $\mathrm{CO}_{2}$ precipitation, and we know that during this time large blocks of $\mathrm{CO}_{2}$ ice also form in order to create the araneiform terrain visible during springtime.

However, their formation processes remain a mystery due to the inability of our passive imaging and spectroscopic instruments to pierce the cloudy enveloping veil. Further, it

\begin{tabular}{|c|c|c|}
\hline Science themes & Measurement objectives & Instrument requirement \\
\hline \multirow{10}{*}{$\begin{array}{l}\text { Science Theme 1. Surface. To detect, map and } \\
\text { quantify deposition of } \mathrm{H}_{2} \mathrm{O} \text { and } \mathrm{CO}_{2} \text { ice during the } \\
\text { polar night }\end{array}$} & $\begin{array}{l}\text { 1. Composition. Differentiate surface } \mathrm{CO}_{2} \text { ice and } \mathrm{H}_{2} \mathrm{O} \\
\text { ice }\end{array}$ & $\begin{array}{l}\text { Use NIR laser DIAL technique to } \\
\text { differentiate ices }\end{array}$ \\
\hline & 2. Grain shape and size. $\mathrm{Map} \mathrm{CO}_{2}$ ice and $\mathrm{H}_{2} \mathrm{O}$ ice grain & Use DIAL and polarization to map \\
\hline & $\begin{array}{l}\text { size/shape properties } \\
\text { 3. Seasonal changes. Map changes in height as ice is }\end{array}$ & $\begin{array}{l}\text { ice properties } \\
\text { Use timed laser returns to create }\end{array}$ \\
\hline & deposited & $\begin{array}{l}\text { high res DTMs to find changes in } \\
\text { snow pack height }\end{array}$ \\
\hline & 4. Seasonal changes. Determine nature of slab ice south & Use NIR laser reflectance DIAL \\
\hline & $\begin{array}{l}\text { cryptic region }[4,8] \text { and re-observe transient "halo" } \\
\text { events [117] }\end{array}$ & $\begin{array}{l}\text { technique to differentiate ices and } \\
\text { polarization to determine } \\
\text { properties }\end{array}$ \\
\hline & 5. Nadir soundings. Monitor thermal cold spot activity & Use NIR laser reflectance DIAL \\
\hline & $\begin{array}{l}\text { and determine whether they are due to } \mathrm{CO}_{2} \text { snow, } \mathrm{CO}_{2} \\
\text { clouds, blizzards or surface ice }[63,74,119]\end{array}$ & $\begin{array}{l}\text { technique to differentiate ices and } \\
\text { polarization to determine }\end{array}$ \\
\hline & & properties \\
\hline & $\begin{array}{l}\text { 6. Surface pressure. Monitor surface pressure and } \\
\text { partial pressure of } \mathrm{H}_{2} \mathrm{O} \text { and produce global, seasonal } \\
\text { maps of surface pressure dynamics }\end{array}$ & $\begin{array}{l}\text { Use NIR laser reflectance DIAL to } \\
\text { derive total atmospheric pressure } \\
\text { and also } \mathrm{H}_{2} \mathrm{O} \text { partial pressure }\end{array}$ \\
\hline \multirow{4}{*}{$\begin{array}{l}\text { Science Theme 2. Ice Clouds. To identify and map } \\
\text { fogs, clouds and cloud properties inside and outside } \\
\text { the polar hood, on a daily basis. }\end{array}$} & $\begin{array}{l}\text { 1. Nadir soundings. Map cloud heights up to } 100 \mathrm{~km} \\
\text { above Martian surface, detect multiple clouds decks }\end{array}$ & $\begin{array}{l}\text { Use NIR reflectance to measure } \\
\text { albedo of cloud ice particles }\end{array}$ \\
\hline & $\begin{array}{l}\text { 2. Composition. Determine cloud compositions and find } \\
\mathrm{CO}_{2}-\mathrm{H}_{2} \mathrm{O} \text { ice clusters }[118]\end{array}$ & $\begin{array}{l}\text { Use NIR multiple channel DIAL } \\
\text { reflectance technique }\end{array}$ \\
\hline & $\begin{array}{l}\text { 3. Grain shape and size. Map cloud particle albedo, size } \\
\text { and orientation }\end{array}$ & $\begin{array}{l}\text { Use polarization to measure albedo } \\
\text { of ice particles }\end{array}$ \\
\hline & $\begin{array}{l}\text { 4. Nadir soundings. Discriminate fogs from } \mathrm{H}_{2} \mathrm{O} \text { ice } \\
\text { deposition on both } \mathrm{CO}_{2} \text { ice caps }[15,16,58]\end{array}$ & $\begin{array}{l}\text { Use timed laser returns to } \\
\text { discriminate low fogs from surface } \\
\text { ice }\end{array}$ \\
\hline \multirow{4}{*}{$\begin{array}{l}\text { Science Theme 3. Dust Aerosols. Map dust storms, } \\
\text { planetary boundary layer, precipitation and aerosol } \\
\text { loads and particle geometries and orientations on a } \\
\text { daily basis }\end{array}$} & $\begin{array}{l}\text { 1. Nadir soundings. Map Planetary Boundary Layer and } \\
\text { aerosol particle heights all mission, determine dust } \\
\text { cloud internal structure and observe multiple decks }\end{array}$ & $\begin{array}{l}\text { Use timed laser returns and } \\
\text { strengths to map aerosols in } \\
\text { atmosphere }\end{array}$ \\
\hline & 2. Grain shape and size. Map dust aerosols properties & $\begin{array}{l}\text { Use NIR polarization to measure } \\
\text { particle properties }\end{array}$ \\
\hline & $\begin{array}{l}\text { 3. Seasonal changes. Map increased dust activity over } \\
\text { south pole geysers }\end{array}$ & $\begin{array}{l}\text { Use timed laser returns and full } \\
\text { Stokes polarization to detect geyser } \\
\text { dynamics and timing }\end{array}$ \\
\hline & $\begin{array}{l}\text { 4. Nadir soundings. Map convective } \mathrm{CO}_{2} \text { cloud towers } \\
\text { [21] }\end{array}$ & $\begin{array}{l}\text { Use polarization to detect particle } \\
\text { orientation/dynamics }\end{array}$ \\
\hline
\end{tabular}

should be noted

that radar

instruments are generally not sensitive to the top $1 \mathrm{~mm}-10 \mathrm{~cm}$ of the ice, where the daily deposition and sublimation processes occur.

Figure 1. ASPEN instrument science themes, measurement objective and instrument requirements [1]. 


\subsection{Previous Lidar Mission - MOLA}

The highly successful Mars Orbiting Laser Altimeter (MOLA) instrument on Mars Global Surveyor measured clouds and the height of the seasonal $\mathrm{CO}_{2}$ surface ice accumulations $[28,29]$. However, its use of a single wavelength $(1.064 \mu \mathrm{m})$ prevented discrimination between $\mathrm{H}_{2} \mathrm{O}$ and $\mathrm{CO}_{2}$ clouds using the MOLA dataset. In addition, MOLA had no ability to assess particle sizes/shapes, nor measure $\mathrm{H}_{2} \mathrm{O}$ or $\mathrm{CO}_{2}$ gas vapor.

The MOLA instrument did demonstrate the ability to detect optically thin Martian dust devils $[30,31]$. Consequently, one can have confidence that ASPEN will be capable of monitoring dust loading and activity, including that associated with the eruption of 'geysers' in the south polar 'Cryptic' Region [8] - because the ASPEN detectors are designed not to saturate over the relatively high albedo Martian ice caps [32].

\subsection{Previous Lidar Mission - Phoenix}

The Phoenix spacecraft landed in the Vastitas Borealis region of Mars (at $68.2^{\circ} \mathrm{N}$ ) in May 2008 and operated for 5 months or 152 Martian days (one summer and fall period) [33]. The Phoenix meteorology station included a vertical pointing Nd:YAG lidar operating at 1.064 and $0.532 \mu \mathrm{m}$. The lidar system successfully detected aerosol structures consistent with Martian cirrus clouds and in particular the 'virga' or "Mare's Tails" (ice particles falling from their formation site in the main cloud deck) as they passed over the lander during the local night [34]. Having no polarization capability, the Phoenix lidar could not directly determine grain shapes. We consider the Phoenix lidar to be a useful pathfinder for future lidar systems such as ASPEN.

\subsection{Previous Terrestrial LIDAR mission - CALIPSO}

The CALIOP laser onboard the Cloud Aerosol Lidar and Infrared Pathfinder Satellite Observations (CALIPSO) space-craft was launched in April 2006 and is still in operation. With an orbit $\sim 700 \mathrm{~km}$, it is part of the 'A-Train' of Earth observing satellites. The CALIOP laser operates at 1.064 and $0.532 \mu \mathrm{m}$, measuring linear polarization in the latter band. The instrument was designed and tested at Ball Aerospace and is operated jointly by NASA and CNES [35].

The surface footprint of the CALIOP is $\sim 100 \mathrm{~m}$ and the vertical resolution is $30-60 \mathrm{~m}$. An example application of the CALIPSO instrument was observation of soot from Arctic wildfires drifting over Greenland [36]. The sensitivity to the aerosols associated with the fires provides a clear demonstration of lidar utility for monitoring/characterizing dust and cloud activity across multiple scales, as well as for studies of low lying fogs and sublimation flow events near the Martian surface. CALIPSO has also been used to 
monitor the height of the terrestrial planetary boundary layer, a useful precursor experiment for ASPEN at Mars. Although CALIOP does not exhibit the same wavelength flexibility and polarimetric capability (i.e. does not measure the full returned Stokes vector) of the ASPEN instrument, its enhanced abilities beyond the MOLA and Phoenix lidar provide further motivation for the concept of an orbital lidar at Mars.

\section{Current TRL and Future technology advancement}

The ASPEN concept is currently at TRL 2-3, with the initial designs and requirements laid out [1] and work on multiwavelength lasers [26]. Work on enabling technologies for variable frequency laser sources and detectors at GSFC has demonstrated the ability of measurement of $\mathrm{CH}_{4}$ in the Earth's atmosphere using a wavelength manifold (rather than individual rotational lines) which has enabled accuracies of $0.5 \%$ [26]. In addition, work continues on a Martian wind-measuring LIDAR, called MARLI [37], which is complementary to the ASPEN concept. The MARLI design is a single wavelength approach that concentrates on atmospheric winds and dust and water ice clouds, in contrast to our multiwavelength, surface and atmosphere approach.

We anticipate a 3 year, $\$ 1$ million project would get the instrument to TRL 4 with a breadboard done and retire much of the risk around the laser and amplifier. To advance the instrument from TRL 4 to TRL 6 , with an instrument in a terrestrial airborne environment, would take roughly \$3-5 million and a further 3-4 years of development. At that stage, the instrument could be proposed for future spaceflight missions.

\section{Application to other planetary bodies}

In this white paper, we have emphasized the utility of the ASPEN lidar instrument for an orbital Mars mission; however the same type of instrument would be applicable for a range of future missions. The multiwavelength polarization sensitive LIDAR would also provide invaluable insights when deployed to other rocky bodies, particularly those with seasonally changing (i.e. insolation driven) plumes of multiple icy volatiles, such as Enceladus, Triton, or Pluto, and insolation driven dust lifting, such as Ceres, Main Belt Comets and the Moon.

The ASPEN instrument concept would be ideal for missions to ice covered bodies (e.g. Europa, Enceladus, Triton, even methane ice on Kuiper Belt objects) to investigate the properties of icy surfaces in low sunlight conditions. As part of a Discovery class mission to active comet-like near Earth asteroids, the system would be ideal for probing the physical properties of a cometary coma. The instrument could also be used in an 
orbital mission to Venus,to probe cloud properties and structure in the NIR windows of the Venusian atmosphere.

\section{Summary}

We have outlined the scientific case for a polarization LIDAR for an eventual orbital mission to Mars. The combination of active, multiple-wavelength measurements with polarimetry makes this instrument concept an essential option in the future inventory of spacecraft instrumentation. The lessons learned from such an instrument would fundamentally advance our understanding of modern day volatile transport and deposition on Mars, and other planetary surfaces with insolation-driven volatile regimes.

\section{References}

[1] Brown A.J. et al. (2015) JQSRT 153 $\underline{131-143}$

[2] Hayne, P.O. et al. (2012) JGR 117 E08014 [3] Brown A.J. et al. (2010) JGR 115 E2, Brown, A.J. et al. (2012) JGR 117 E12 [4] Wolff M.J. et al. (2010) Icarus 208 143-55 [5] Langevin Y. et al. (2006) Nature 442 790-2 [6] Appere, T. et al. (2011) JGR 116, E05001 [7] Titus, T.N. et al. (2003) Science 299 1048-51

[8] Kieffer, H.H. and Titus, T.N. (2001) Icarus $201154 \underline{162-80}$

[9] Kieffer, H.H. (2006) Nature 442 793-6 [10] Brown, A.J. et al. (2016) Icarus 277401 [11] NASA (2006). Solar System Exploration 2006 Solar System Exploration Roadmap. [12] Calvin W, et al. (2007) Report from the 2013 Mars Science Orbiter (MSO) SAG [13] Gary-Bicas, C.E. et al. (2020) JGR $125 \underline{5}$ [14] Langevin, Y. et al. (2007) JGR 112 EE [15] Inada, A. (2007) Icarus 192 378-95 [16] Titus, T.N. (2005) GRL $32 \underline{24}$ [17] Forget, F. and Pollack, J.B. (1996) JGR $101 \underline{16865}$

[18] Hayne, P. et al. (2013) Icarus $231 \underline{122-30}$ [19] Piqueux, S. and Christensen P.R. (2008) JGR 113 E6 [20] Colaprete, A. et al. (2003) JGR 108 E7
[21] Ma Y. et al. (2011) JQSRT $112 \underline{338-45}$ [22] Lu, X. et al. (2010) JQSRT 112 320-8

[23] Raack et al. (2020) Icarus $350 \underline{113889}$ Dundas, et al. (2019) GSL $467 \underline{67}$ [24] Diniega, S. et al. (2010) Geology 1047 Diniega, S. et al. (2020) GSL $467 \underline{95}$ [25] Michaels, T. (2006) GRL $33 \underline{\lfloor 16201}$ Rafkin, S. (2011) PSS 112 147-154 Heavens, NG, et al. (2019) JAS $\underline{3299-3326}$ [26] Riris, H. et al. (2020) SPIE abstract [27] Riris, H. et al. (2019) CEAS Space J [28] Smith DE, et al. (1999) Science $284 \underline{1495}$ [29] Pettengill $G$ and Ford P (2000) GRL 27:609

[30] Ivanov AB and Muhleman DO. (2001) Icarus 154 190-206

[31] Neumann GA, et al. (2003) JGR 2003;108 E⿱ E4

[32] Heavens, N.G. (2017) Icarus 289 1-21

[33] Smith PH, et al. (2009) Science 325

58-61.

[34] Whiteway JA, et al. (2009) Science 325

68-70.

[35] Weimer CA, (2007) vol. 6555, San Diego, CA: SPIE.

[36] Box J, et al. (2012), AGU Fall abstract C51E-03 San Francisco, CA

[37] Cremons,D. et al (2020) CEAS SJ $12 \underline{149}$ 